\title{
Numerical Simulations of the Hall-Petch Relationship in Aluminium Using Gradient-Enhanced Plasticity Model
}

\author{
Yooseob Song, ${ }^{1}$ Jaeheum Yeon ${ }^{(D)},{ }^{2}$ and Byoungjoon $\mathrm{Na}^{3}$ \\ ${ }^{1}$ Department of Civil Engineering, The University of Texas Rio Grande Valley, Edinburg, TX 78539, USA \\ ${ }^{2}$ Department of Engineering \& Technology, Texas A\&M University-Commerce, Commerce, TX 75429, USA \\ ${ }^{3}$ Research Institute for Mega Construction, Korea University, Seoul 02841, Republic of Korea
}

Correspondence should be addressed to Jaeheum Yeon; jyeon81@tamu.edu

Received 9 August 2019; Accepted 15 November 2019; Published 5 December 2019

Academic Editor: Mario D’Aniello

Copyright (c) 2019 Yooseob Song et al. This is an open access article distributed under the Creative Commons Attribution License, which permits unrestricted use, distribution, and reproduction in any medium, provided the original work is properly cited.

The Hall-Petch relation in aluminium is discussed based on the strain gradient plasticity framework. The thermodynamically consistent gradient-enhanced flow rules for bulk and grain boundaries are developed using the concepts of thermal activation energy and dislocation interaction mechanisms. It is assumed that the thermodynamic microstresses for bulk and grain boundaries have dissipative and energetic contributions, and in turn, both dissipative and energetic material length scale parameters are existent. Accordingly, two-dimensional finite element simulations are performed to analyse characteristics of the Hall-Petch strengthening and the Hall-Petch constants. The proposed flow rules for the grain boundary are validated using the existing experimental data from literatures. An excellent agreement between the numerical results and the experimental measurements is obtained in the Hall-Petch plot. In addition, it is observed that the Hall-Petch constants do not remain unchanged but vary depending on the strain level.

\section{Introduction}

Most of the metals and metal alloys have polycrystalline nature. In general, a fine-grained material is stronger and harder than a coarse-grained one. This can be described in the relation between the grain size and yield stress through the Hall-Petch equation as follows $[1,2]$ :

$$
\sigma_{y}=\sigma_{0}+\frac{k}{\sqrt{D}}
$$

where $\sigma_{y}$ denotes the yield stress, $\sigma_{0}$ denotes the material constant related to the resistance of lattice to dislocation motion, $k$ denotes the Hall-Petch strengthening coefficient, and $D$ denotes the average grain size. A linear relationship between $\sigma_{y}$ and $D^{-1 / 2}$ with a slope of $k$ is shown in the Hall-Petch plot.

After the pioneering works of Hall [1] for mild steels and Petch [2] for brittle materials, numerous works have been conducted to investigate the Hall-Petch relation through various methods including experiments [3], review/overview
$[4,5]$, theoretical investigations [6], and numerical simulations [7]. In [3], microhardness of nanocrystalline palladium and copper was experimentally investigated according to grain size variation, and significant increases in strength were observed in both materials compared with conventional grain size materials. The predictive capability of the Hall-Petch relation as well as its physical basis was discussed briefly by [4], based on experimental findings. Connections of the Hall-Petch relation to strain rate sensitivities; shear banding; fracture mechanics; fatigue; hardness property; broader stress-strain behaviour of hexagonal close packed, bodycentered cubic, and face-centered cubic materials; and ductile-to-brittle transition behaviour of steel and related materials were reported in [5]. Pande and Cooper [6] focused on the inverse Hall-Petch relation, which manifests itself as the softening of nanocrystalline materials of very small mean grain sizes. In [7], the strain gradient crystal plasticity theory and its finite element algorithm were developed to describe the grain size-dependent behaviours of polycrystalline materials. 
Even in these days, the Hall-Petch relation is of great interest to many researchers. Yu et al. [8] presented a review of the Hall-Petch relationship in magnesium alloys, especially focusing on the Hall-Petch slope $(k)$ and the factors influencing the mechanisms of $k$. The Hall-Petch relationship in Al-ZnO composites with different matrix grain sizes $\left(D_{1}\right)$ relative to interparticle spacing $\left(D_{2}\right)$ was studied in [9]. For samples with $D_{1}>D_{2}$, it was observed that both particle strengthening mechanism and the grain size effects described by the Hall-Petch relationship contribute to the strengthening of the metal matrix composites. In [10], the Hall-Petch breakdown in nanocrystalline ceramics was tested by performing indentation studies on fully dense nanocrystalline ceramics fabricated with grain sizes ranging from 3.6 to $37.5 \mathrm{~nm}$. It was observed that the maximum hardness occurs at a grain size of $18.4 \mathrm{~nm}$, and the inverse (or negative) Hall-Petch relationship reduces the hardness as the grain size is decreased to roughly $5 \mathrm{~nm}$.

There are several numerical studies on the Hall-Petch strengthening based on crystal plasticity [11]. However, strain gradient continuum plasticity is rarely used in this area. Voyiadjis and coworkers [12-19] have developed the coupled thermo-mechanical and thermodynamically consistent strain gradient plasticity models to study the characteristics of nano/microscale metallic materials. In this work, strain gradient-enhanced flow rules for bulk and grain boundaries are proposed to investigate the grain size-dependent flow stress of polycrystalline materials.

Therefore, the main aim of this work is to show that the proposed strain gradient-enhanced flow rules for bulk and grain boundaries well capture the Hall-Petch relation.

\section{Gradient-Enhanced Continuum Plasticity}

In the current work, the subscripts $i, j, k, l, m$, and $n$ are used to denote tensors. The superscripts "dis," "en," "ext," "int," "GB," "e," and "p" stand for specific quantities such as dissipative, energetic, external, internal, grain boundary, elastic state, and plastic state, respectively.

2.1. Principle of Virtual Power. The external power $\mathbb{P}^{\text {ext }}$ expended by the macrotraction $\mathbb{T}_{i}$ and microtraction $m$ on the external surface $\partial \Omega_{0}$ and the generalized external body force $b_{i}$ acting in $\Omega_{0}$ as follows $[15,18]$ :

$$
\mathbb{P}^{\mathrm{ext}}=\int_{\Omega_{0}} b_{i} \dot{u}_{i} \mathrm{~d} V+\int_{\partial \Omega_{0}}\left(t_{i} \dot{u}_{i}+m \dot{\varepsilon}^{\mathrm{p}}+a \dot{T}\right) \mathrm{d} S,
$$

where $\dot{u}_{i}$ denotes the macroscopic velocity. The term $\varepsilon^{\mathrm{p}}$ denotes the accumulated plastic strain, and the terms $T$ and $\dot{T}$ denote the temperature and its rate, respectively. In addition, for the thermal effect, term $a$ is present in the external power.

In the arbitrary region $\Omega_{0}$, the internal power $\mathbb{P}^{\text {int }}$ is assumed as follows with a combination of macroenergy, microenergy, and thermal energy contributions $[15,18]$ :

$$
\mathbb{P}^{\mathrm{int}}=\int_{\Omega_{0}}\left(\sigma_{i j} \dot{\varepsilon}_{i j}^{\mathrm{e}}+\xi \dot{\varepsilon}^{\mathrm{p}}+\mathbb{Q}_{i} \dot{\varepsilon}_{i}^{\mathrm{p}}+\mathbb{A} \dot{T}+\mathbb{B}_{i} \dot{T}_{, i}\right) \mathrm{d} V,
$$

where $\varepsilon_{i j}^{\mathrm{e}}$ is the elastic part of the strain tensor, $\xi$ and $\mathbb{Q}_{i}$ are the thermodynamic microforces conjugate, respectively, to $\dot{\varepsilon}^{\mathrm{p}}$, and $\dot{\varepsilon}_{i,}^{\mathrm{p}}, \mathbb{A}$, and $\mathbb{B}_{i}$ are the micromorphic scalar and vector generalized stresses conjugate to the temperature rate $\dot{T}$ and the gradient of the temperature rate $\dot{T}_{, i}$, respectively, and $\sigma_{i j}$ is the Cauchy stress tensor.

From the relation, $\mathbb{P}^{\text {ext }}=\mathbb{P}^{\text {int }}$ and the divergence theorem, the balance equations in volume $\Omega_{0}$ are obtained, respectively, as follows:

$$
\begin{aligned}
\sigma_{i j, j}+b_{i} & =0, \\
\bar{\sigma}_{i j} & =\left(\xi-\mathbb{Q}_{k, k}\right) N_{i j}, \\
\mathbb{B}_{i, i}-\mathbb{A} & =0,
\end{aligned}
$$

where $\bar{\sigma}_{i j}$ denotes the deviatoric part of $\sigma_{i j}, \bar{\sigma}_{i j}=$ $\sigma_{i j}-\sigma_{k k} \delta_{i j} / 3$, where $\delta_{i j}$ denotes the Kronecker delta. Equation (4) represents the macroscopic linear momentum balance equation and the nonlocal microforce balance equation, respectively. The term $N_{i j}$ denotes the direction of plastic flow given by $N_{i j}=\dot{e}_{i j}^{\mathrm{p}} / \hat{\varepsilon}^{\mathrm{p}}$.

The local and nonlocal traction balance equations on $\partial \Omega_{0}$ are given as follows:

$$
\begin{aligned}
t_{j} & =\sigma_{i j} n_{i}, \\
m & =\mathbb{Q}_{i} n_{i}, \\
a & =\mathbb{B}_{i} n_{i},
\end{aligned}
$$

where $n_{i}$ denotes the outward unit vector normal to $\partial \Omega_{0}$.

Next, a thermodynamically consistent grain boundary flow rule is developed in this work. Consider the two grains $\mathscr{G}_{1}$ and $\mathscr{G}_{2}$ separated by the grain boundary. The continuous displacement field across the grain boundary is assumed, $u_{i}^{G_{1}}=u_{i}^{\mathscr{G}_{2}}$. It is also assumed on the arbitrary surface $S^{\mathrm{GB}}$ over the grain boundary that the grain boundary internal virtual power depends on the grain boundary accumulated plastic strain rates $\dot{\varepsilon}^{\mathrm{F}^{\mathrm{GB} \mathscr{G}_{1}}}$ at $S^{\mathrm{GB} \mathscr{G}_{1}}$ and $\dot{\varepsilon}^{\mathrm{p}^{\mathrm{GB} \mathscr{E}_{2}}}$ at $S^{\mathrm{GB} \mathscr{G}_{2}}[16,18]$ :

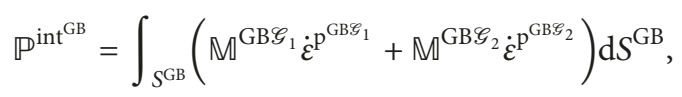

where the grain boundary micromoment tractions $\mathbb{M}^{\mathrm{GB} \mathscr{G}_{1}}$ and $\mathbb{M}^{\mathrm{GB} \mathscr{G}_{2}}$ are assumed to, respectively, expend power over $\dot{\mathcal{E}}^{\mathrm{P}^{\mathrm{GB} \mathscr{G}_{1}}}$ and $\dot{\mathcal{\varepsilon}}^{\mathrm{p}^{\mathrm{GB} \mathscr{G}_{2}}}$. The external power $\mathbb{P}^{\text {ext }}{ }^{\mathrm{GB}}$ is expended by the macrotractions $\sigma_{i j}^{\mathscr{G}_{1}}\left(-n_{j}^{\mathrm{GB}}\right)$ and $\sigma_{i j}^{\mathscr{G}_{2}}\left(n_{j}^{\mathrm{GB}}\right)$ and the microtractions $\mathbb{Q}_{k}^{\mathscr{C}_{1}}\left(-n_{k}^{\mathrm{GB}}\right)$ and $\mathbb{Q}_{i}^{\mathscr{G}_{2}}\left(n_{k}^{\mathrm{GB}}\right)$ as follows $[16,18]$ :

$$
\begin{aligned}
\mathbb{P}^{\mathrm{ext}^{\mathrm{GB}}}= & \int_{S^{\mathrm{GB}}}\left\{\left(\sigma_{i j}^{\mathscr{G}_{2}} n_{j}^{\mathrm{GB}}-\sigma_{i j}^{\mathscr{I}_{1}} n_{j}^{\mathrm{GB}}\right) \dot{u}_{i}+\mathbb{Q}_{k}^{\mathscr{G}_{2}} n_{k}^{\mathrm{GB}} \dot{\mathcal{E}}^{\mathrm{p}^{\mathrm{GB} \mathscr{S}_{2}}}\right. \\
& \left.-\mathbb{Q}_{k}^{\mathscr{C}_{1}} n_{k}^{\mathrm{GB}} \dot{\varepsilon}^{\mathrm{E}^{\mathrm{GB} \mathscr{G}_{1}}}\right\} \mathrm{d} S^{\mathrm{GB}},
\end{aligned}
$$

where $\mathbf{n}^{\mathrm{GB}}$ denotes the unit vector normal to the grain boundary surface. Using $\mathbb{P}^{\text {int }}{ }^{\mathrm{GB}}=\mathbb{P}^{\text {ext }}{ }^{\mathrm{GB}}$, the grain boundary macroscopic and microscopic force balance equations can be obtained as 


$$
\begin{aligned}
\left(\sigma_{i j}^{\mathscr{I}_{1}}-\sigma_{i j}^{\mathscr{G}_{2}}\right) n_{j}^{\mathrm{GB}} & =0, \\
\mathbb{M}^{\mathrm{GB} \mathscr{G}_{1}}+\mathbb{Q}_{k}^{\mathscr{G}_{1}} n_{k}^{\mathrm{GB}} & =0, \\
\mathbb{M}^{\mathrm{GB} \mathscr{G}_{2}}-\mathbb{Q}_{k}^{\mathscr{G}_{2}} n_{k}^{\mathrm{GB}} & =0 .
\end{aligned}
$$

2.2. Thermodynamic Microforces: Energetic and Dissipative Components. The Helmholtz free energy $\Psi$ is given through the Legendre transform as $\Psi=\mathscr{E}-T \mathfrak{s}$, where $\mathscr{E}$ denotes the internal energy and $s$ denotes the entropy [20]. Using this relation, the following Clausius-Duhem inequality is constructed [15]:

$$
\sigma_{i j} \dot{\varepsilon}_{i j}^{\mathrm{e}}+\xi \dot{\varepsilon}^{\mathrm{p}}+\mathbb{Q}_{i} \dot{\varepsilon}_{, i}^{\mathrm{p}}+\mathbb{A} \dot{T}+\mathbb{B}_{i} \dot{T}_{, i}-\rho \dot{\Psi}-\rho s \dot{T}-q_{i} \frac{T_{, i}}{T} \geq 0 .
$$

In order to take the effect of nonuniform distribution of microdefects into account along with temperature on the homogenized material behaviour, it is assumed that the Helmholtz free energy is a smooth function of $\varepsilon_{i j}^{\mathrm{e}}, \varepsilon^{\mathrm{p}}, \varepsilon_{, i}^{\mathrm{p}}, T$, and $T_{, i}$. During the process of deriving the constitutive equations, it is important to make sure that nonnegative dissipation is maintained. It should be mentioned that the Helmholtz free energy is at its minimum in a stable equilibrium state with respect to any isothermal small geometrically admissible virtual displacement field. In this regard, different counterparts of $\Psi$ are locally convex functions of $\varepsilon_{i j}^{\mathrm{e}}, \varepsilon^{\mathrm{p}}$, and $\varepsilon_{, i}^{\mathrm{p}}$ at all points of the body in the considered equilibrium state, and $\Psi$ is a concave function of temperature [15].

The thermodynamic microforces $\xi, \mathbb{Q}_{i}$, and $\mathbb{A}$ are assumed to have the energetic and dissipative contributions $[12-16,18]$. Thus, $\xi=\xi^{\text {en }}+\xi^{\text {dis }}, \mathbb{Q}_{i}=\mathbb{Q}_{i}^{\text {en }}+\mathbb{Q}_{i}^{\text {dis }}$, and $\mathbb{A}=\mathbb{A}^{\text {en }}+\mathbb{A}^{\text {dis }}$. From the Clausius-Duhem inequality and the aforementioned decompositions, the energetic microforces are defined as

$$
\begin{aligned}
\sigma_{i j} & =\rho \frac{\partial \Psi}{\partial \varepsilon_{i j}^{\mathrm{e}}}, \\
\xi^{\mathrm{en}} & =\rho \frac{\partial \Psi}{\partial \varepsilon^{\mathrm{p}}}, \\
\mathbb{Q}_{i}^{\mathrm{en}} & =\rho \frac{\partial \Psi}{\partial \varepsilon_{, i}^{\mathrm{p}}}, \\
\mathbb{A}^{\text {en }} & =\rho\left(s+\frac{\partial \Psi}{\partial T}\right), \\
\mathbb{B}_{i} & =\rho \frac{\partial \Psi}{\partial T_{, i}} .
\end{aligned}
$$

The dissipative microstresses are then determined from the dissipation potential $\mathscr{D}\left(\dot{\mathcal{\varepsilon}}^{\mathrm{p}}, \dot{\varepsilon}_{, i}^{\mathrm{p}}, \dot{T}, T_{, i}\right)$ as follows:

$$
\begin{aligned}
\xi^{\text {dis }} & =\frac{\partial \mathscr{D}}{\partial \dot{\varepsilon}^{\mathrm{p}}}, \\
\mathbb{Q}_{i}^{\mathrm{dis}} & =\frac{\partial \mathscr{D}}{\partial \dot{\varepsilon}_{, i}^{\mathrm{p}}}, \\
\mathbb{A}^{\mathrm{dis}} & =\frac{\partial \mathscr{D}}{\partial \dot{T}}, \\
-\frac{q_{i}}{T} & =\frac{\partial \mathscr{D}}{\partial T_{, i}} .
\end{aligned}
$$

Similarly, the grain boundary energetic and dissipative microforces are defined as

$$
\begin{aligned}
& \mathbb{M}^{\mathrm{GB}, \text { en }}=\rho \frac{\partial \Psi^{\mathrm{GB}}}{\partial \mathcal{\varepsilon}^{\mathrm{GB}}}, \\
& \mathbb{M}^{\mathrm{GB}, \mathrm{dis}}=\frac{\partial \mathscr{D}^{\mathrm{GB}}}{\partial \dot{\varepsilon}^{\mathrm{GB}}},
\end{aligned}
$$

where the grain boundary Helmholtz free energy $\left(\Psi^{\mathrm{GB}}\right)$ is a function of $\varepsilon^{\mathrm{p}^{\mathrm{GB}}}$, i.e., $\Psi^{\mathrm{GB}}=\Psi^{\mathrm{GB}}\left(\varepsilon^{\mathrm{p}^{\mathrm{GB}}}\right)$ and the grain boundary thermodynamic microforce quantitity $\mathbb{M}^{\mathrm{GB}}$ has the energetic and dissipative contributions, i.e., $\mathbb{M}^{\mathrm{GB}}=\mathbb{M}^{\mathrm{GB} \text {,en }}+\mathbb{M}^{\mathrm{GB} \text {,dis }}$. The components $\mathbb{M}^{\mathrm{GB} \text {,en }}$ and $\mathbb{M}^{\mathrm{GB} \text {,dis }}$ are related to the preslip and postslip transfer mechanisms; therefore, the grain boundary accumulated plastic strains for the preslip transfer

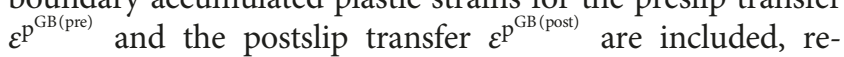
spectively, $\left(\varepsilon^{\mathrm{p}^{\mathrm{GB}}}=\varepsilon^{\mathrm{p}^{\mathrm{GB}(\text { pre })}}+\varepsilon^{\mathrm{p}^{\mathrm{GB}(\text { post) }}}\right)$. $\mathscr{D}^{\mathrm{GB}}$ denotes the nonnegative grain boundary dissipation density per unit time $\mathscr{D}^{\mathrm{GB}}=\mathbb{M}^{\mathrm{GB}, \mathrm{dis}} \dot{\mathcal{E}}^{\mathrm{p}} \geq 0$. This nonnegative plastic dissipation condition is satisfied in the case that $\mathscr{D}^{G B}$ is a convex function of $\dot{\mathcal{E}}^{\mathrm{p}^{\mathrm{GB}}}$.

2.3. Energetic and Dissipative Constitutive Relations. The Helmholtz free energy function in this work is assumed based on $[12,13,18]$ as follows:

$$
\begin{aligned}
\Psi= & \frac{1}{2 \rho} \varepsilon_{i j}^{\mathrm{e}} E_{i j k l} \varepsilon_{k l}^{\mathrm{e}}+\frac{h_{0}}{\rho\left(r^{\mathrm{p}}+1\right)}\left[1-\left(\frac{T}{T_{y}}\right)^{n}\right]\left(\varepsilon^{\mathrm{p}}\right)^{\mu+1} \\
& +\frac{\sigma_{*}}{\rho(\vartheta+1)}\left[\ell_{\mathrm{en}}^{2}\left(\varepsilon_{, i}^{\mathrm{p}} \varepsilon_{, i}^{\mathrm{p}}\right)\right]^{(9+1) / 2}-\frac{1}{2} \frac{c_{\varepsilon}}{T_{r}}\left(T-T_{r}\right)^{2} \\
& -\frac{\alpha^{\mathrm{th}}}{\rho}\left(T-T_{r}\right) \varepsilon_{i j}^{\mathrm{e}} \delta_{i j}-\frac{1}{2 \rho} a T_{, i} T_{, i},
\end{aligned}
$$

where $E_{i j k l}$ denotes the elastic modulus tensor, $\alpha^{\text {th }}$ denotes the thermal expansion coefficient, $h_{0}$ and $r$ denote the material parameters related to isotropic hardening, $T_{y}$ and $n$ denote the material parameters related to the thermal effects, $\sigma_{*}>0$ denotes the initial slip resistance scaling parameter, $\ell_{\text {en }}$ denotes the energetic material length scale, $a$ denotes the material parameter related to the isotropic heat conduction, $\vartheta$ denotes the material parameter related to the nonlinearity of the defect energy, $c_{\varepsilon}$ denotes the specific heat capacity at the constant stress, and $T_{r}$ denotes the reference temperature. 
From equations (10) and (13), the energetic microforces can be obtained as follows:

$$
\begin{aligned}
\sigma_{i j}= & E_{i j k l} \varepsilon_{k l}^{\mathrm{e}}-\alpha^{\mathrm{th}}\left(T-T_{r}\right) \delta_{i j}, \\
\xi^{\mathrm{en}}= & h_{0}\left[1-\left(\frac{T}{T_{y}}\right)^{n}\right]\left(\varepsilon^{\mathrm{p}}\right)^{\mu}, \\
\mathbb{Q}_{i}^{\mathrm{en}}= & \sigma_{*} \ell_{\mathrm{en}}^{2}\left[\ell_{\mathrm{en}}^{2}\left(\varepsilon_{, k}^{\mathrm{p}} \varepsilon_{, k}^{\mathrm{p}}\right)\right]^{(9-1) / 2} \varepsilon_{, i}^{\mathrm{p}}, \\
\mathbb{A}^{\mathrm{en}}= & \rho J-\frac{c_{\varepsilon}}{T_{r}}\left(T-T_{r}\right)-\alpha^{\mathrm{th}}\left(T-T_{r}\right) \varepsilon_{i j}^{\mathrm{e}} \delta_{i j} \\
& -\frac{h_{0}\left(\varepsilon^{\mathrm{p}}\right)^{\mu+1}}{\gamma^{\mathrm{r}}+1} \frac{T}{T_{y}}\left(\frac{T}{T_{y}}\right)^{n-1}, \\
\mathbb{B}_{i}= & -a T_{, i} .
\end{aligned}
$$

The dissipation potential has the following functional form in this work $[12,13,18]$ :

$$
\begin{aligned}
\mathscr{D}= & \sigma_{*} \sqrt{\mathscr{H}^{2}\left(\varepsilon^{\mathrm{p}}\right)+\ell_{\mathrm{NG}} e^{\mathrm{p}}}\left[1-\left(\frac{T}{T_{y}}\right)^{n}\right]\left(\frac{\dot{\varepsilon}^{\mathrm{p}}}{\dot{\mathcal{R}}_{1}}\right)^{m_{1}} \dot{\mathcal{E}}^{\mathrm{p}} \\
& +\sigma_{*}\left[1-\left(\frac{T}{T_{y}}\right)^{n}\right]\left(\frac{\dot{R}}{\dot{\mathcal{R}}_{2}}\right)^{m_{2}} \dot{p}-\frac{\varsigma}{2} \dot{T}^{2}-\frac{1}{2} \frac{k(T)}{T} T_{, i} T_{, i},
\end{aligned}
$$

where $\dot{R}_{1}>0$ and $\dot{R}_{2}>0$ denote the reference rate parameters, $m_{1}>0$ and $m_{2}>0$ denote the rate sensitivity parameters, $\varsigma$ denotes the material parameter related to the energy exchange between electron and phonon, and $k(T)$ denotes the thermal conductivity coefficient. $\ell_{\mathrm{NG}}$ denotes the NG (Nix-Gao) material length scale firstly introduced by Nix and Gao [21]. When $\mathscr{H}\left(\varepsilon^{\mathrm{p}}\right)=1$ and $\ell_{\mathrm{NG}}=0$, equation (15) reduces to the one used in [12]. $e^{\mathrm{p}}$ is defined as $e^{\mathrm{p}} \stackrel{\text { def }}{=}\left\|\alpha_{i j}\right\|=b \rho_{G}$, where $\alpha_{i j}$ denotes the Nye dislocation density tensor, $b$ denotes the magnitude of the Burgers vector, and $\rho_{G}$ denotes the density of geometrically necessary dislocations.

The scalar $\dot{p}$ measures the gradient of plastic strain rate, and it is defined as $\dot{\mathcal{R}} \stackrel{\text { def }}{=} \ell_{\text {dis }}\left\|\dot{\varepsilon}_{, i}^{\mathrm{p}}\right\|=\ell_{\text {dis }} \sqrt{\dot{\varepsilon}_{, i}^{\mathrm{p}} \dot{\varepsilon}_{, i}^{\mathrm{p}}}$, where $\ell_{\text {dis }}$ denotes the dissipative material length scale $[12,13,18]$.

The strain hardening/softening behaviour is determined through the dimensionless function $\mathscr{H}\left(\varepsilon^{\mathrm{p}}\right)$. The following mixed-form hardening function is considered in this work [22]:

$$
\mathscr{H}\left(\varepsilon^{\mathrm{p}}\right)=1+(\chi-1)\left[1-\exp \left(-\omega \mathcal{E}^{\mathrm{p}}\right)\right]+\frac{h_{0}}{\sigma_{*}} \mathcal{\varepsilon}^{\mathrm{p}},
$$

where $\omega$ and $\chi$ denote the material constants.

Using equations (11) and (15) along with the assumption $\nprec(T) / T=k_{0}=$ constant, the dissipative microforces can be obtained as follows:

$$
\begin{aligned}
\xi^{\mathrm{dis}} & =\sigma_{*} \sqrt{\mathscr{H}^{2}\left(\varepsilon^{\mathrm{p}}\right)+\ell_{\mathrm{NG}} e^{\mathrm{p}}}\left[1-\left(\frac{T}{T_{y}}\right)^{n}\right]\left(\frac{\dot{\varepsilon}^{\mathrm{p}}}{\dot{\mathcal{R}}_{1}}\right)^{m_{1}}, \\
\mathbb{Q}_{i}^{\mathrm{dis}} & =\sigma_{*} \ell_{\mathrm{dis}}^{2}\left(m_{2}+1\right)\left[1-\left(\frac{T}{T_{y}}\right)^{n}\right]\left(\frac{\dot{\mathcal{R}}}{\dot{\mathcal{R}}_{2}}\right)^{m_{2}} \frac{\dot{\varepsilon}_{, i}^{\mathrm{p}}}{\dot{\dot{R}}} \\
\mathbb{A}^{\text {dis }} & =-\varsigma \dot{T}, \\
\frac{\mathfrak{q}_{i}}{T} & =k_{0} T_{, i} .
\end{aligned}
$$

The grain boundary free energy per unit surface $\Psi^{\mathrm{GB}}$ in this work is assumed to have the general power law form as follows [23]:

$$
\Psi^{\mathrm{GB}}\left(\varepsilon^{\mathrm{p}^{\mathrm{GB}}}\right)=\frac{1}{2} G \ell_{\mathrm{en}}^{\mathrm{GB}}\left(\varepsilon^{\mathrm{p}^{\mathrm{GB}(\mathrm{pre})}}\right)^{2},
$$

where $G$ denotes the shear modulus and $\ell_{\mathrm{en}}^{\mathrm{GB}}$ denotes the grain boundary energetic length scale. From equations (12) and (18), the grain boundary energetic microforce is determined as

$$
\mathbb{M}^{\mathrm{GB}, \mathrm{en}}=G \ell_{\mathrm{en}}^{\mathrm{GB}} \mathcal{E}^{\mathrm{p}^{\mathrm{GB}(\mathrm{pre})}} .
$$

The generalized expression of the grain boundary dissipation potential is put forward in this work as follows $[14,16]$ :

$$
\begin{aligned}
\mathscr{D}^{\mathrm{GB}}= & \frac{\ell_{\mathrm{dis}}^{\mathrm{GB}}}{m^{\mathrm{GB}}+1}\left(\sigma_{*}^{\mathrm{GB}}+h_{0}^{\mathrm{GB}} \mathcal{E}^{\mathrm{p}^{\mathrm{GB}(\text { post })}}\right)\left(1-\frac{T^{\mathrm{GB}}}{T_{y}^{\mathrm{GB}}}\right)^{n^{\mathrm{GB}}} \\
& \cdot\left(\frac{\dot{\mathcal{\varepsilon}}^{\mathrm{p}^{\mathrm{GB}(\text { post })}}}{\dot{\mathcal{R}}^{\mathrm{GB}}}\right)^{m^{\mathrm{GB}}} \dot{\mathcal{\varepsilon}}^{\mathrm{p}^{\mathrm{GB}(\text { post })}} \geq 0,
\end{aligned}
$$

where $\ell_{\mathrm{dis}}^{\mathrm{GB}}$ denotes the grain boundary dissipative length scale, $m^{\mathrm{GB}}$ and $\dot{p}^{\mathrm{GB}}$ denote the material parameters related to viscosity, $\sigma_{*}^{\mathrm{GB}}$ is the stress-dimensioned parameter related to the grain boundary yield stress, $h_{0}^{\mathrm{GB}}$ denotes the grain boundary hardening constant, $T_{y}^{\mathrm{GB}}$ denotes the grain boundary thermal constant at the onset of yielding, $\mathbf{n}^{\mathrm{GB}}$ denotes the grain boundary thermal constant. The rate and temperature dependency of the grain boundary energy are presented through the terms $\left(\dot{\varepsilon}^{\mathrm{p}^{\mathrm{GB}(\text { post })}} / \dot{p}^{\mathrm{GB}}\right)^{m^{\mathrm{GB}}}$ and $\left(1-T^{\mathrm{GB}} / T_{y}^{\mathrm{GB}}\right)^{\mathbf{n}^{\mathrm{GB}}}$, respectively.

Substitution of equation (20) into equation (12) gives the grain boundary dissipative microforce $\mathbb{M}^{\mathrm{GB} \text {,dis }}$ as follows:

$$
\mathbb{M}^{\mathrm{GB}, \mathrm{dis}}=\ell_{\mathrm{dis}}^{\mathrm{GB}}\left(\sigma_{*}^{\mathrm{GB}}+h_{0}^{\mathrm{GB}} \varepsilon^{\mathrm{p}^{\mathrm{GB}(\text { post })}}\right)\left(1-\frac{T^{\mathrm{GB}}}{T_{y}^{\mathrm{GB}}}\right)^{\mathbf{n}^{\mathrm{GB}}}\left(\frac{\dot{\varepsilon}^{\mathrm{p}^{\mathrm{GB}(\text { post })}}}{\dot{p}^{\mathrm{GB}}}\right)^{m^{\mathrm{GB}}} .
$$

Finally, the grain boundary thermodynamic microforce $\mathbb{M}^{\mathrm{GB}}$ is obtained by combining equations (19) and (21) as follows: 


$$
\begin{aligned}
\mathbb{M}^{\mathrm{GB}}= & G \ell_{\mathrm{en}}^{\mathrm{GB}} \mathcal{E}^{\mathrm{p}^{\mathrm{GB}(\text { pre })}}+\ell_{\mathrm{dis}}^{\mathrm{GB}}\left(\sigma_{*}^{\mathrm{GB}}+h_{0}^{\mathrm{GB}} \varepsilon^{\mathrm{p}^{\mathrm{GB}(\text { post })}}\right) \\
& \cdot\left(1-\frac{T^{\mathrm{GB}}}{T_{y}^{\mathrm{GB}}}\right)^{\mathbf{n}^{\mathrm{GB}}}\left(\frac{\dot{\mathcal{\varepsilon}}^{\mathrm{GB}(\text { post })}}{\dot{p}^{\mathrm{GB}}}\right)^{m^{\mathrm{GB}}} \cdot
\end{aligned}
$$

From equation (22), it is obvious that the grain boundary acts like free surface when microscopically free boundary condition is imposed $\left(\ell_{\mathrm{en}}^{\mathrm{GB}}=\ell_{\mathrm{dis}}^{\mathrm{GB}}=0\right)$, while passivated condition at the grain boundary can be described when the microscopically hard boundary condition is imposed $\left(\ell_{\mathrm{en}}^{\mathrm{GB}} \longrightarrow \infty\right.$ and $\left.\ell_{\mathrm{dis}}^{\mathrm{GB}} \longrightarrow \infty\right)$.

2.4. Flow Rules. One can establish the flow rule from the nonlocal microforce balance, equation (4), and the energetic and dissipative microforce quantities. The backstress is considered in this work in the microforce equilibrium, i.e., $\bar{\sigma}_{i j}-\left(-\mathbb{Q}_{k, k}^{\mathrm{en}}\right) N_{i j}=\left(\xi-\mathbb{Q}_{k, k}^{\mathrm{dis}}\right) N_{i j}$, where $N_{i j}=\dot{e}_{i j}^{\mathrm{p}} / \dot{\mathcal{E}}^{\mathrm{p}}$. The following flow rule for the bulk can be obtained [12, 13, 15].

$$
\begin{aligned}
\bar{\sigma}_{i j} & -\left[-\sigma_{*} \ell_{\mathrm{en}}^{2}\left\{\ell_{\mathrm{en}}^{2}\left(\varepsilon_{, i}^{\mathrm{p}} \varepsilon_{, i}^{\mathrm{p}}\right)\right\}^{(9-1) / 2} \varepsilon_{, k k}^{\mathrm{p}}\right] N_{i j}=\left[h_{0}\left[1-\left(\frac{T}{T_{y}}\right)^{n}\right]\left(\varepsilon^{\mathrm{p}}\right)^{\mu}\right. \\
& +\sigma_{*} \sqrt{\mathscr{H}^{2}\left(\varepsilon^{\mathrm{p}}\right)+\ell_{\mathrm{NG}} \mathrm{e}^{\mathrm{p}}}\left[1-\left(\frac{T}{T_{y}}\right)^{n}\right]\left(\frac{\dot{\varepsilon}^{\mathrm{p}}}{\dot{p}_{1}}\right)^{m_{1}} \\
& \left.-\sigma_{*} \ell_{\mathrm{dis}}^{2}\left(m_{2}+1\right)\left[1-\left(\frac{T}{T_{y}}\right)^{n}\right]\left(\frac{\dot{p}}{\dot{p}_{2}}\right)^{m_{2}} \frac{\dot{\varepsilon}_{, k k}^{\mathrm{p}}}{\dot{p}}\right] N_{i j} .
\end{aligned}
$$

The substitution of equation (22) into the grain boundary microforce balances, equation (8), gives the grain boundary flow rules as follows $[16,18]$ :

For $S^{\mathrm{GB} \mathscr{G}_{1}}$,

$$
\begin{aligned}
& {\left[\sigma_{*} \ell_{\mathrm{en}}^{2}\left[\ell_{\mathrm{en}}^{2}\left(\varepsilon_{, k}^{\mathrm{p}} \mathcal{E}_{, k}^{\mathrm{p}}\right)\right]^{(9-1) / 2} \varepsilon_{, i}^{\mathrm{p}}+\sigma_{*} \ell_{\mathrm{dis}}^{2}\left(m_{2}+1\right)\right.} \\
& \left.\left[1-\left(\frac{T}{T_{y}}\right)^{n}\right]\left(\frac{\dot{p}}{\dot{p}_{2}}\right)^{m_{2}} \frac{\left.\dot{\varepsilon}_{, i}^{\mathrm{p}}\right]}{\dot{p}}\right] n_{k}^{\mathrm{GB}}+G \ell_{\mathrm{en}}^{\mathrm{GB}} \varepsilon^{\mathrm{p}^{\mathrm{GB}(\mathrm{pre})}} \\
& =-\ell_{\mathrm{dis}}^{\mathrm{GB}}\left(\sigma_{*}^{\mathrm{GB}}+h_{0}^{\mathrm{GB}} \varepsilon^{\mathrm{p}^{\mathrm{GB}(\text { post })}}\right)\left(1-\frac{T^{\mathrm{GB}}}{T_{y}^{\mathrm{GB}}}\right)^{n^{\mathrm{GB}}}\left(\frac{\dot{\varepsilon}^{\mathrm{p}^{\mathrm{GB}(\text { post })}}}{\dot{p}^{\mathrm{GB}}}\right)^{m^{\mathrm{GB}}} .
\end{aligned}
$$

For $S^{\mathrm{GB} \mathscr{G}_{2}}$,

$$
\begin{aligned}
& {\left[\sigma_{*} \ell_{\text {en }}^{2}\left[\ell_{\text {en }}^{2}\left(\varepsilon_{, k}^{\mathrm{p}} \varepsilon_{, k}^{\mathrm{p}}\right)\right]^{(9-1) / 2} \varepsilon_{, i}^{\mathrm{p}}+\sigma_{*} \ell_{\mathrm{dis}}^{2}\left(m_{2}+1\right)\right.} \\
& {\left[1-\left(\frac{T}{T_{y}}\right)^{n}\right]\left(\frac{\dot{p}}{\dot{\mathcal{R}}_{2}}\right)^{m_{2} \dot{\varepsilon}_{, i}^{\mathrm{p}}} \frac{\dot{\dot{R}}}{\dot{p}} n_{k}^{\mathrm{GB}}-G \ell_{\mathrm{en}}^{\mathrm{GB}} \varepsilon^{\mathrm{p}} \mathrm{p}^{\mathrm{GB}(\text { pre })}} \\
& =\ell_{\mathrm{dis}}^{\mathrm{GB}}\left(\sigma_{*}^{\mathrm{GB}}+h_{0}^{\mathrm{GB}} \varepsilon^{\mathrm{p}^{\mathrm{GB}(\text { post })}}\right)\left(1-\frac{T^{\mathrm{GB}}}{T_{y}^{\mathrm{GB}}}\right)^{\mathrm{n}^{\mathrm{GB}}}\left(\frac{\dot{\varepsilon}^{\mathrm{p}^{\mathrm{GB}(\text { post })}}}{\dot{p}^{\mathrm{GB}}}\right)^{m^{\mathrm{GB}}},
\end{aligned}
$$

where the second terms in left-hand side of equations (24) and (25) indicate the backstress. The grain boundary flow rules, equations (24) and (25), are only applied for the nodes on the grain boundaries.

The developed flow rules for the bulk and grain boundaries are numerically implemented through the finite element simulations to address the microstructural material characteristics. In this work, the unknown nodal degrees of freedom are the displacement field $u_{i}$ and the plastic strain field $\varepsilon^{\mathrm{p}}$, and they are independently discretized.

\section{Model Validation and Calibration of the Model Parameters}

The proposed strain gradient-dependent flow rules will be validated in this section through the comparison against the experimental measurements by [24]. The calibration of some model parameters will also be carried out simultaneously. In [24], the Bauschinger effect in sputter-deposited aluminium (Al) thin film was investigated experimentally. However, the Bauschinger effect will not be studied in this work since it is not of interest; instead, the experimental data from passivated and unpassivated layers will be used for the model validation.

The sample preparation method is introduced in [24] in detail. Samples are vacuum-annealed at $300^{\circ} \mathrm{C}$ for stabilization of the material microstructure. It is observed through transmission electron microscope (TEM) micrographs that the average grain size is $2.1 \mu \mathrm{m}$.

The material parameters for $\mathrm{Al}$ are also calibrated using the experimental measurements. Tables 1 and 2 show the general and calibrated material parameters, respectively. As mentioned earlier, the grain boundary can behave like a free surface through the microscopically free boundary condition, under $\ell_{\mathrm{en}}^{\mathrm{GB}}=\ell_{\mathrm{dis}}^{\mathrm{GB}}=0$, whereas the passivated condition on film surfaces can be described with $\ell_{\mathrm{en}}^{\mathrm{GB}} \longrightarrow \infty$ and $\ell_{\mathrm{dis}}^{\mathrm{GB}} \longrightarrow \infty$. Stress-strain responses of passivated and unpassivated films from experiments and simulations are shown in Figure 1. As clearly shown in this figure, the numerical results and experimental measurements correspond with each other closely.

\section{Numerical Results: Hall-Petch Relation}

Materials can be strengthened by decreasing the average grain size. This method is called grain boundary strengthening or Hall-Petch strengthening. Grain boundary impedes the movements of dislocations and how many dislocations are existent in a grain affects on how smoothly they can travel from grain to grain. Hall-Petch strengthening is based on this observation. In this section, HallPetch strengthening is investigated using finite element simulations with different grain sizes based on the proposed model.

4.1. Problem Description. The schematic illustration of uniaxial strain problem with single-crystal and polycrystalline materials is shown in Figure 2. The problem 
TABLE 1: General material parameters of aluminium for the current numerical simulations $[15,18]$.

\begin{tabular}{lc}
\hline General material parameters & Values \\
\hline$E(\mathrm{GPa})$ & 70 \\
$\nu$ & 0.30 \\
$\mu(\mathrm{GPa})$ & 27 \\
$\rho\left(\mathrm{g} \cdot \mathrm{cm}^{-3}\right)$ & 2.702 \\
$c_{\varepsilon}\left(\mathrm{J} / \mathrm{g} \cdot{ }^{\circ} \mathrm{K}\right)$ & 0.910 \\
$\alpha^{\mathrm{th}}\left(\mu \mathrm{m} / \mathrm{m} \cdot{ }^{\circ} \mathrm{K}\right)$ & 24.0 \\
$\dot{p}_{1}, \dot{p}_{2}\left(s^{-1}\right)$ & 0.04 \\
$r$ & 0.6 \\
$m_{1}$ & 0.05 \\
$m_{2}$ & 0.2 \\
$T_{y}\left({ }^{\circ} \mathrm{K}\right)$ & 933 \\
$n$ & 0.3 \\
\hline
\end{tabular}

TABle 2: Calibrated material parameters of aluminium for the current numerical simulations.

\begin{tabular}{lc}
\hline Calibrated material parameters & Values \\
\hline$\sigma_{*}(\mathrm{MPa})$ & 100 \\
$h_{0}(\mathrm{MPa})$ & 100 \\
$\ell_{\mathrm{en}}(\mu \mathrm{m})$ & 1.0 \\
$\ell_{\mathrm{dis}}(\mu \mathrm{m})$ & 2.5 \\
$\ell_{\mathrm{NG}}(\mu \mathrm{m})$ & 1.0 \\
\hline
\end{tabular}

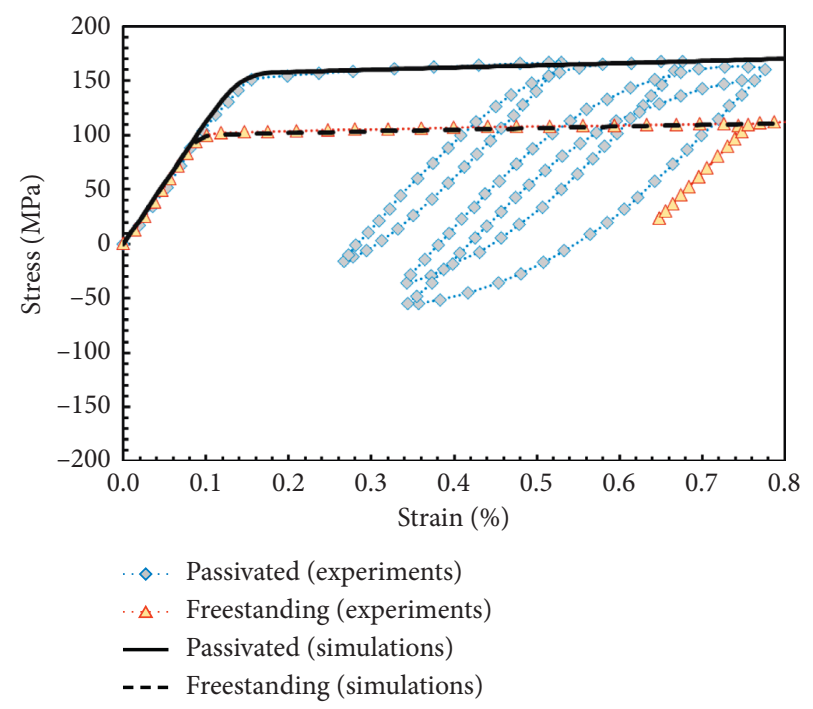

Figure 1: Proposed model validation using the experimental data in [24] on the stress-strain responses in aluminium thin films.

geometry, initial condition, loading condition, macroscopic boundary condition, and finite element mesh are displayed in this figure. The term $u^{\dagger}(t)$ represents the prescribed displacement. Each grain has an average grain size of $D$. The whole square is split into several grains by grain boundaries, which are represented by bold lines, as shown in Figure 2. $4096(64 \times 64)$ elements are used. The general material parameters in Table 1 are used, while the rest of the material parameters are calibrated using another set of experiments for pure aluminium $(99.999 \%)$ by [25]. Since the experiments were performed for grain sizes from 0.035 to $1.3 \mathrm{~mm}$ by [25], six different grain sizes, $D=0.03125 \mathrm{~mm}$ $(32 \times 32=1024$ grains $), \quad D=0.0625 \mathrm{~mm} \quad(16 \times 16=256$ grains $), D=0.125 \mathrm{~mm}(8 \times 8=$

64 grains $), D=0.25 \mathrm{~mm}(4 \times 4=16$ grains $), D=0.5 \mathrm{~mm}$ $(2 \times 2=4$ grains), and $D=1.0 \mathrm{~mm}$ (single grain) are considered in the current simulations. In this work, room temperature is assumed as is in [25].

4.2. Grain Boundary Simulations. To check that the proposed grain boundary flow rules in equations (24) and (25) can properly mimic the two null conditions (free surface and passivated surface), two simulations are performed in single crystal with the microscopically free boundary condition and the microscopically hard boundary condition at the grain boundary. Figure 3 shows the distributions of the accumulated plastic strain $\varepsilon^{\mathrm{p}}$ with the microscopically free boundary condition and the microscopically hard boundary condition. As expected, uniform distribution of $\varepsilon^{\mathrm{p}}$ is observed in case of the microscopically free boundary condition like free surface, while total blockage of dislocation movement at the grain boundary $\left(\varepsilon^{\mathrm{p}}=0\right)$ is well described by imposing the microscopically hard boundary condition in the proposed grain boundary flow rules.

4.3. Hall-Petch Strengthening. Numerical simulations under the uniaxial tensile loading condition are carried out with six different grain sizes from 0.03125 to $1.0 \mathrm{~mm}$ to investigate the Hall-Petch strengthening and to validate the proposed model by comparing with the experimental data of [25]. The Hall-Petch constants such as $\sigma_{0}$ and $k$ are also studied in this section. The numerically obtained true stress-true strain curves are shown in Figure 4 with varying grain sizes. As can be seen in this figure, the Hall-Petch strengthening (material hardens with the decreasing grain size) is well observed qualitatively.

The yield stress at a (true) strain of 0.002 and the flow stresses at four selected strains $(0.01,0.05,0.1$, and 0.2 ) against the reciprocal square root of the grain size are plotted in Figure 5. Straight lines are fitted to the points by the least square method. A significant grain size effect is predicted at the onset of yielding, which is in line with experimental finding. Both experimental data [25] and model predictions show a good linear correlation for all the grain sizes. Moreover, model predictions from the simulations are in an excellent agreement with experimental data. Table 3 shows the Hall-Petch constants, $\sigma_{0}$ and $k$, for both experiments and simulations at the different levels of strain obtained from the linear trendlines in Figure 5. Again, simulations show a good agreement with experiments in both parameters.

Figure 5 and Table 3 imply that the Hall-Petch constants, $\sigma_{0}$ and $k$, increase with increasing strain. It is worth mentioning that the strengthening effect of the grain boundary areas described by $k$ increases with increasing strain.

These two parameters are plotted in Figure 6 as a function of strain. Besides the presented experimental data at the five strain levels in Figure 5 and Table 3, the other experimental datasets presented in [25] are also plotted in 


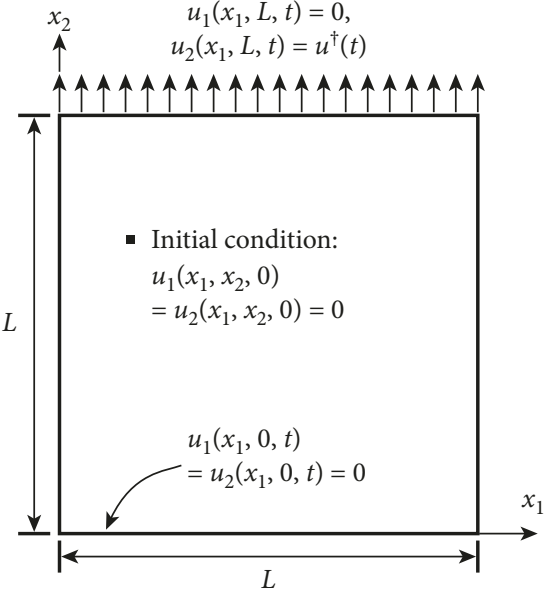

(a)

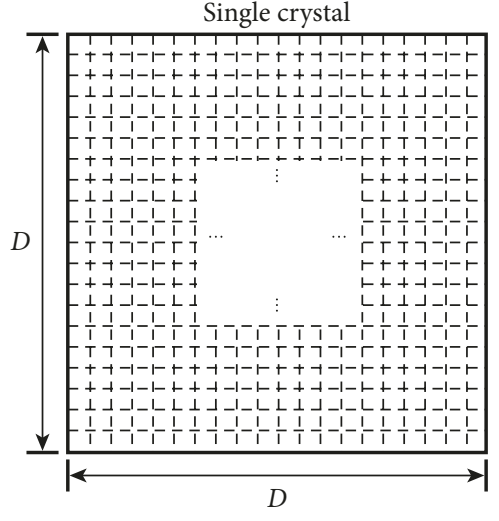

(b)

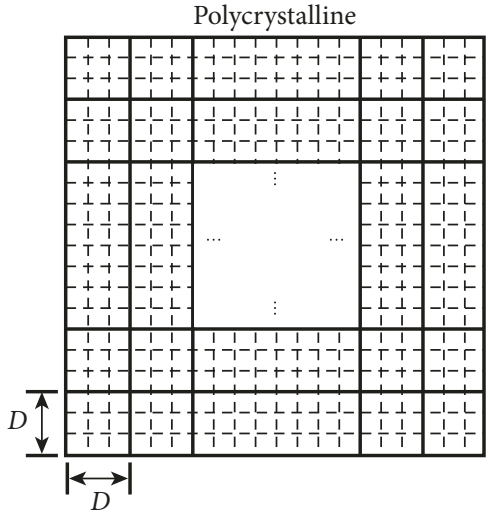

(c)

FIGURE 2: Schematic illustration of uniaxial strain problem with single crystal and polycrystalline materials. $D$ is the (average) grain size. Dotted lines and bold lines in single crystal and polycrystalline materials represent finite element mesh and grain boundary, respectively.

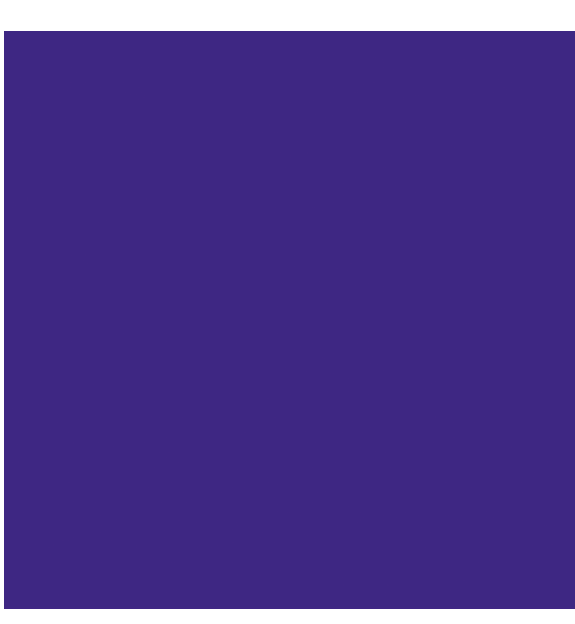

(a)

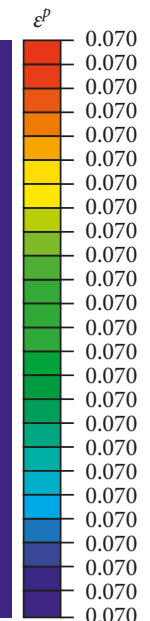

0.070
0.070

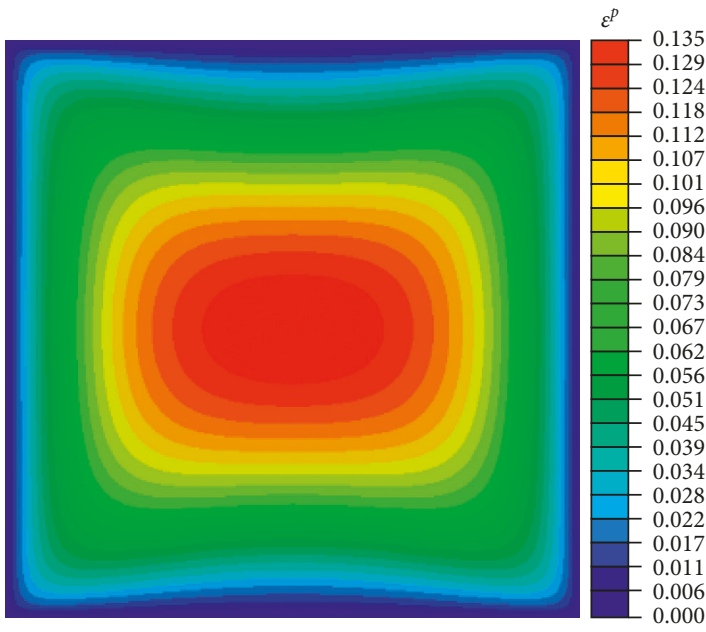

(b)

FiguRE 3: Distributions of the accumulated plastic strain in single crystal with (a) microscopically free boundary condition and (b) microscopically hard boundary condition $[15,18]$.

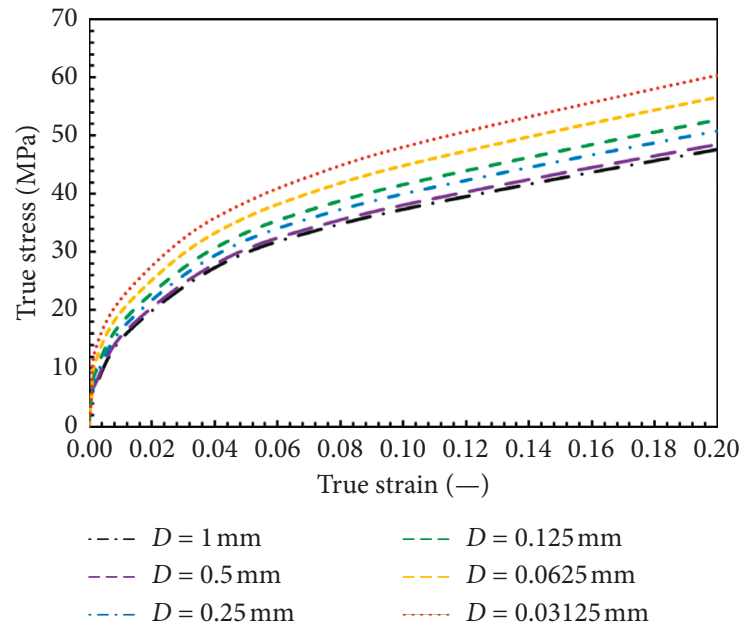

FIgURE 4: True stress-true strain responses with varying grain sizes from simulations. 


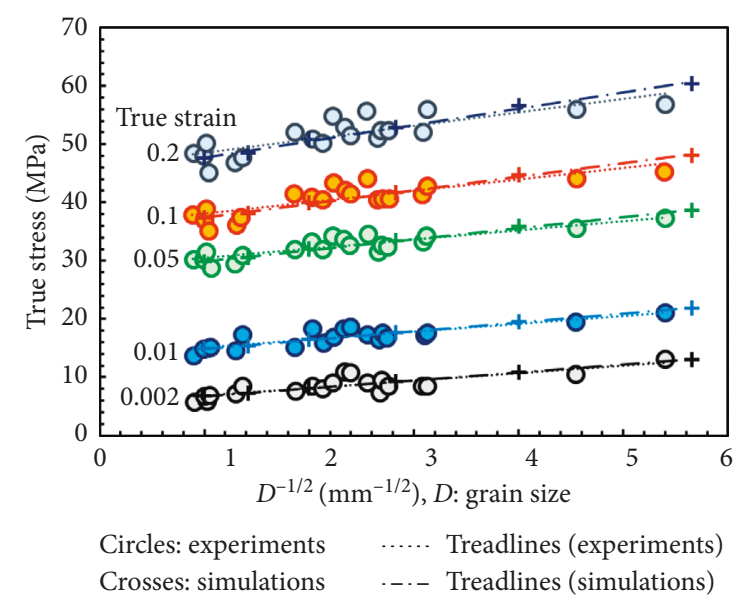

Figure 5: Hall-Petch plot for pure aluminium from experiments [25] and simulations.

TABle 3: Hall-Petch constants for pure aluminium from experiments [25] and simulations.

\begin{tabular}{lcccc}
\hline $\begin{array}{l}\text { True } \\
\text { strain }\end{array}$ & \multicolumn{2}{c}{$\begin{array}{c}\text { Experiments [25] } \\
\sigma_{0}(\mathrm{MPa})\end{array}$} & $k\left(\mathrm{MPa} \cdot \mathrm{mm}^{1 / 2}\right)$ & \multicolumn{2}{c}{ Simulations } \\
& $\sigma_{0}(\mathrm{MPa})$ & $k\left(\mathrm{MPa} \cdot \mathrm{mm}^{1 / 2}\right)$ \\
\hline 0.002 & 5.62 & 1.27 & 5.39 & 1.37 \\
0.01 & 13.83 & 1.39 & 13.37 & 1.49 \\
0.05 & 28.92 & 1.58 & 28.05 & 1.70 \\
0.1 & 36.15 & 1.95 & 35.07 & 2.15 \\
0.2 & 46.32 & 2.29 & 44.94 & 2.60 \\
\hline
\end{tabular}

this figure. The good correlations between experiments and simulations are obtained.

This is in line with the findings of [26]. In [26], an empirical relationship of Hall [1] and Petch [2], equation (1), is extended by expressing the Hall-Petch constants, $\sigma_{0}$ and $k$, to depend on the strain level, such that $\sigma_{y}=\sigma_{0}(\varepsilon)+k(\varepsilon) D^{-n}$, where the exponent $n$ typically ranges from 0.3 to 1.0 (the most reported value is 0.5 ).

Due to the formation of dislocation pile-ups at grain boundaries, flow stress can be enhanced and yielding occurs when flow stress is large enough to cause the slip to propagate from one grain to the adjacent grain. In order to underpin this behaviour, physically based strain gradient plasticity models are in demand. The current model is phenomenological, and physically based models have not been attained despite its importance. Furthermore, the pioneering measurements made by [27] were reported without assessment in terms of the Hall-Petch constants, $\sigma_{*}$ and $k$, but nevertheless show on examination decreasing values of both parameters with increase in temperature, more so in $\sigma_{*}$. A weak strain rate dependence of the flow stress was also measured by [27]. Effects of temperature and strain rate on the Hall-Petch constants will be investigated in the future. In addition, strain gradient crystal plasticity is another interest of the authors so that its pros and cons compared with the strain gradient continuum plasticity covered in this work will be explored in the future work.

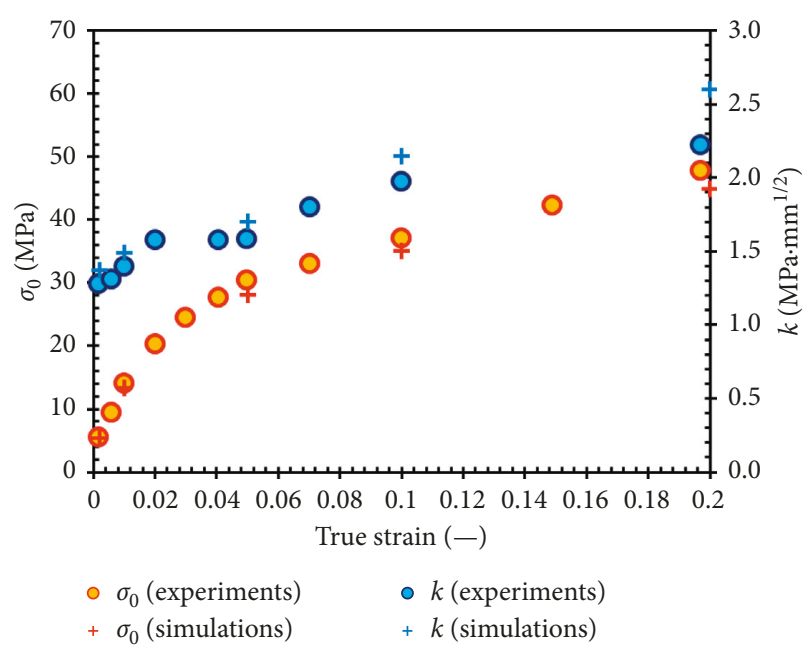

FIGURE 6: Hall-Petch constants as a function of strain for pure aluminium from experiments [25] and simulations.

\section{Conclusions}

The Hall-Petch relation and its strengthening effects on the flow stress of deformed metals were investigated based on the strain gradient plasticity model. The thermodynamically consistent strain gradient dependent plasticity flow rules for bulk and grain boundaries were developed. The proposed theory was implemented through the finite element simulations. Through the comparison with the existing experimental data in the literature, the model proposed was validated.

To study the Hall-Petch relation and characteristics of the grain boundary, the uniaxial strain problem was solved. The two null boundary conditions at the grain boundary, the microscopically free and hard boundary conditions, were well captured through the proposed grain boundary flow rule. The six different grain sizes were considered in this work, and the Hall-Petch equation was well described. The good correlations between the simulated results and the experimental measurements were presented at the five selected strain levels. Lastly, it was clearly observed that the Hall-Petch constants change according to the variation of strain level.

\section{Data Availability}

The data used to support the findings of this study are available from the corresponding author upon request.

\section{Conflicts of Interest}

The authors declare that there are no conflicts of interest regarding the publication of this paper.

\section{References}

[1] E. O. Hall, "The deformation and ageing of mild steel: III discussion of results," Proceedings of the Physical Society. Section B, vol. 64, no. 9, pp. 747-753, 1951. 
[2] N. J. Petch, "The cleavage strength of polycrystals," Journal of the Iron and Steel Institute, vol. 174, pp. 25-28, 1953.

[3] J. R. Weertman, "Hall-Petch strengthening in nanocrystalline metals," Materials Science and Engineering: A, vol. 166, no. 12, pp. 161-167, 1993.

[4] N. Hansen, "Hall-Petch relation and boundary strengthening," Scripta Materialia, vol. 51, no. 8, pp. 801-806, 2004.

[5] R. W. Armstrong, "60 years of Hall-Petch: past to present nano-scale connections," Materials Transactions, vol. 55, no. 1, pp. 2-12, 2014.

[6] C. S. Pande and K. P. Cooper, "Nanomechanics of Hall-Petch relationship in nanocrystalline materials," Progress in Materials Science, vol. 54, no. 6, pp. 689-706, 2009.

[7] L. P. Evers, D. M. Parks, W. A. M. Brekelmans, and M. G. D. Geers, "Crystal plasticity model with enhanced hardening by geometrically necessary dislocation accumulation," Journal of the Mechanics and Physics of Solids, vol. 50, no. 11, pp. 2403-2424, 2002.

[8] H. Yu, Y. Xin, M. Wang, and Q. Liu, "Hall-Petch relationship in Mg alloys: a review," Journal of Materials Science \& Technology, vol. 34, no. 2, pp. 248-256, 2018.

[9] C. L. Li, Q. S. Mei, J. Y. Li, F. Chen, Y. Ma, and X. M. Mei, "Hall-Petch relations and strengthening of Al-ZnO composites in view of grain size relative to interparticle spacing," Scripta Materialia, vol. 153, pp. 27-30, 2018.

[10] H. Ryou, J. W. Drazin, K. J. Wahl et al., "Below the Hall-Petch limit in nanocrystalline ceramics," ACS Nano, vol. 12, no. 4, pp. 3083-3094, 2018.

[11] W. A. Counts, M. V. Braginsky, C. C. Battaile, and E. A. Holm, "Predicting the Hall-Petch effect in fcc metals using non-local crystal plasticity," International Journal of Plasticity, vol. 24, no. 7, pp. 1243-1263, 2008.

[12] G. Z. Voyiadjis and Y. Song, "Effect of passivation on higher order gradient plasticity models for non-proportional loading: energetic and dissipative gradient components," Philosophical Magazine, vol. 97, no. 5, pp. 318-345, 2017.

[13] G. Z. Voyiadjis, Y. Song, and T. Park, "Higher-order thermomechanical gradient plasticity model with energetic and dissipative components," Journal of Engineering Materials and Technology, vol. 139, no. 2, 2017.

[14] Y. Song, A Coupled Thermo-Mechanical Theory of Strain Gradient Plasticity for Small and Finite Deformations, Louisiana State University, Baton Rouge, LA, USA, 2018.

[15] Y. Song and G. Z. Voyiadjis, "Small scale volume formulation based on coupled thermo-mechanical gradient enhanced plasticity theory," International Journal of Solids and Structures, vol. 134, pp. 195-215, 2018.

[16] Y. Song and G. Z. Voyiadjis, "A two-dimensional finite element model of the grain boundary based on thermo-mechanical strain gradient plasticity," Journal of Theoretical and Applied Mechanics, vol. 56, pp. 377-391, 2018.

[17] G. Z. Voyiadjis and Y. Song, "Higher order thermo-mechanical gradient plasticity model: non-proportional loading with energetic and dissipative components," in Handbook of Nonlocal Continuum Mechanics for Materials and Structures, G. Z. Voyiadjis, Ed., Springer International Publishing, Cham, Switzerland, 2017.

[18] G. Z. Voyiadjis and Y. Song, "Finite element analysis of thermodynamically consistent strain gradient plasticity theory and applications," in Handbook of Nonlocal Continuum Mechanics for Materials and Structures, pp. 1-58, Springer, Cham, Switzerland, 2018.

[19] G. Z. Voyiadjis and Y. Song, "Strain gradient continuum plasticity theories: theoretical, numerical and experimental investigations," International Journal of Plasticity, vol. 121, pp. 21-75, 2019.

[20] M. E. Gurtin, E. Fried, and L. Anand, The Mechanics and Thermodynamics of Continua, Cambridge University Press, Cambridge, UK, 2010.

[21] W. D. Nix and H. Gao, "Indentation size effects in crystalline materials: a law for strain gradient plasticity," Journal of the Mechanics and Physics of Solids, vol. 46, no. 3, pp. 411-425, 1998.

[22] E. Voce, "A practical strain-hardening function," Metallurgica, vol. 51, pp. 219-226, 1955.

[23] P. Fredriksson and P. Gudmundson, "Competition between interface and bulk dominated plastic deformation in strain gradient plasticity," Modelling and Simulation in Materials Science and Engineering, vol. 15, no. 1, pp. S61-S69, 2007.

[24] Y. Xiang and J. J. Vlassak, "Bauschinger effect in thin metal films," Scripta Materialia, vol. 53, no. 2, pp. 177-182, 2005.

[25] N. Hansen, "The effect of grain size and strain on the tensile flow stress of aluminium at room temperature," Acta Metallurgica, vol. 25, no. 8, pp. 863-869, 1977.

[26] R. Armstrong, I. Codd, R. M. Douthwaite, and N. J. Petch, "The plastic deformation of polycrystalline aggregates," The Philosophical Magazine: A Journal of Theoretical Experimental and Applied Physics, vol. 7, no. 73, pp. 45-58, 1962.

[27] R. P. Carreker and W. R. Hibbard, "Tensile deformation of aluminum as a function of temperature, strain rate, and grain size," JOM, vol. 9, no. 10, pp. 1157-1163, 1957. 


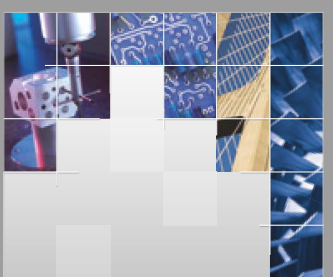

\section{Enfincering}
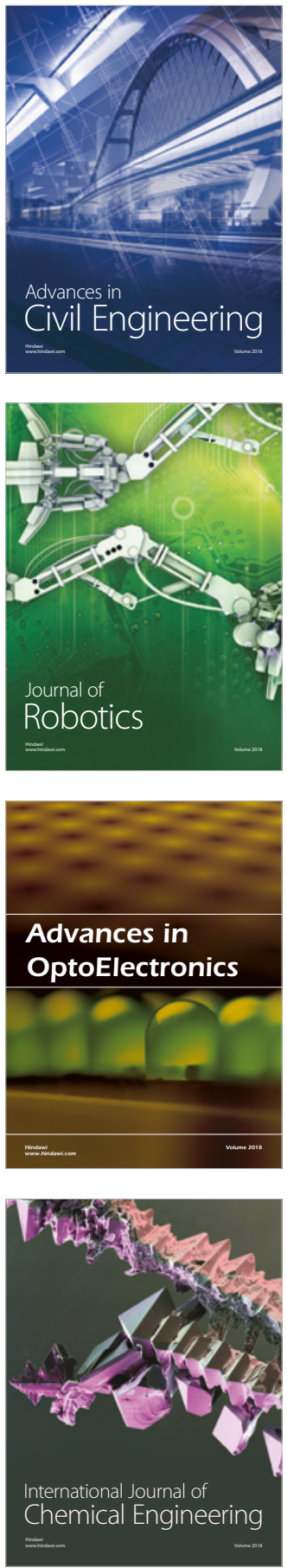

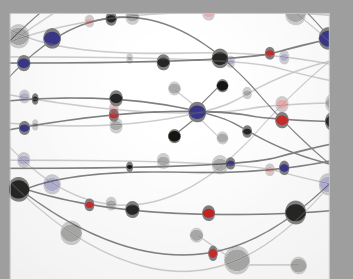

\section{Rotating \\ Machinery}

The Scientific World Journal

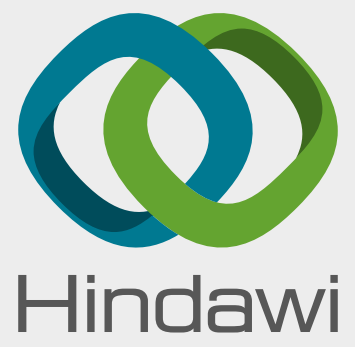

Submit your manuscripts at

www.hindawi.com
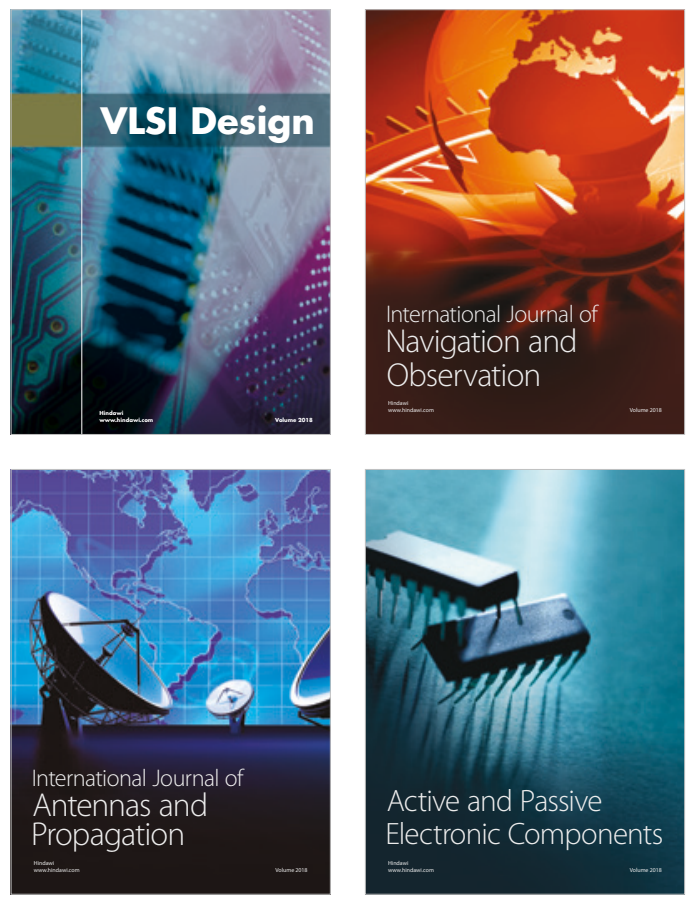
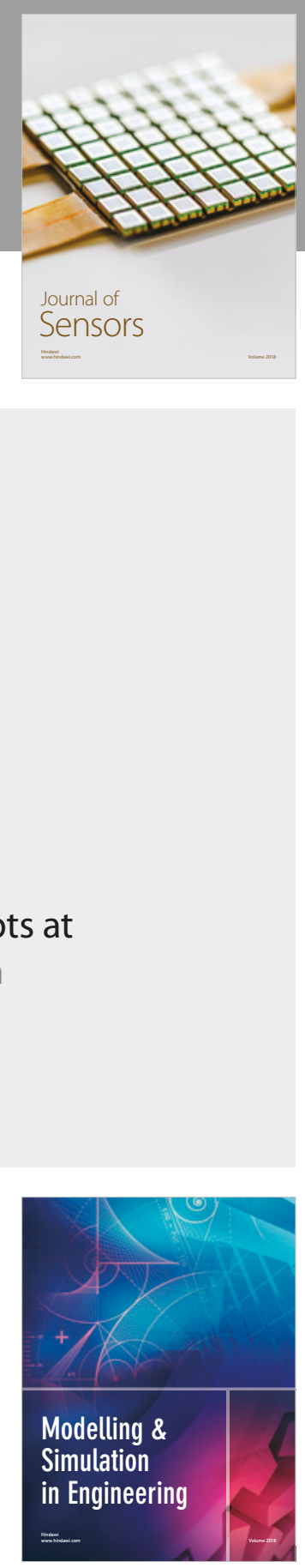

\section{Advances \\ Multimedia}
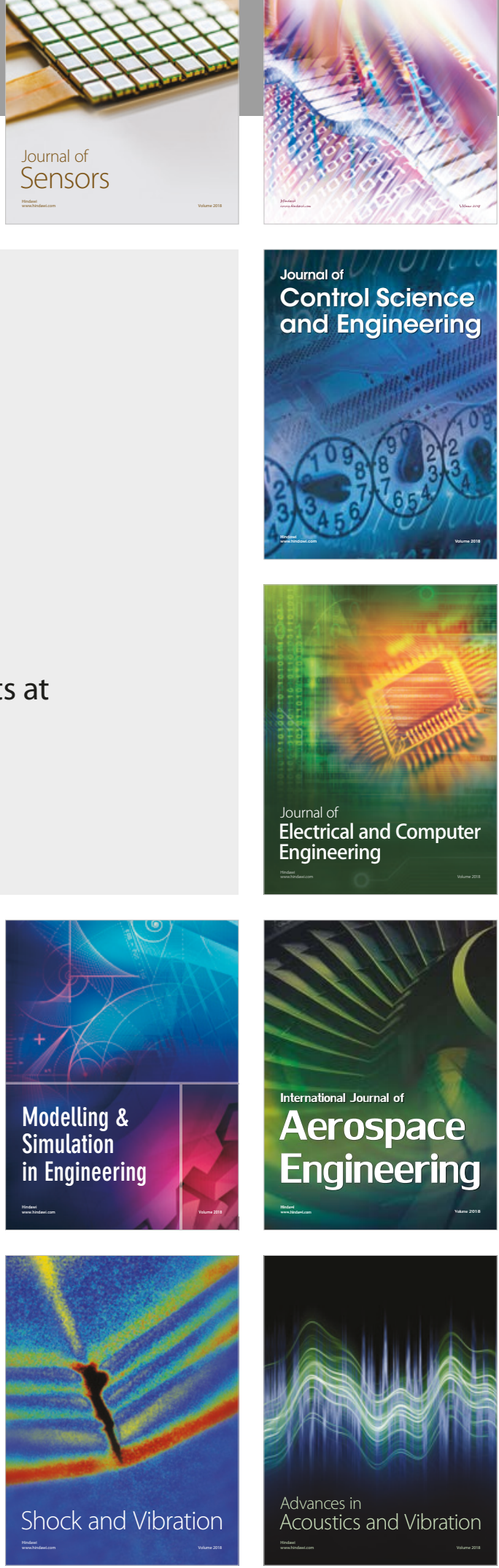